\title{
Pacific
}

Journal of

Mathematics

\section{ON INFINITESIMAL BEHAVIOR OF THE KOBAYASHI DISTANCE}

\author{
MYUNG YULL PANG
}




\title{
ON INFINITESIMAL BEHAVIOR OF THE KOBAYASHI DISTANCE
}

\author{
MyUng-Yull PANG
}

\begin{abstract}
The condition that the Kobayashi distance between two nearby points in a pseudo-convex domain is realized by the Poincaré distance on a single analytic disk joining the two points is studied. It is shown that the condition forces the Kobayashi indicatrix to be convex. Examples of pseudo-convex domains on which this condition fails to hold are given. The (infinitesimal) Kobayashi metric is shown to be a directional derivative of the Kobayashi distance. It is shown that, if the condition holds near any point of a pseudo-convex domain and if the Kobayashi metric is a complete Finsler metric of class $C^{2}$, then the Kobayashi distance between any two points in the domain can be realized by the Poincaré distance on a single analytic disk joining the two points.
\end{abstract}

1. Introduction. In this paper, we study the infinitesimal behavior of the Kobayashi distance on a pseudo-convex domain. In particular, we examine the condition that the distance between two nearby points in a pseudo-convex domain is realized by the Poincare distance on a single analytic disk joining the two points.

Let $D$ be a bounded domain in $\mathbf{C}^{m}$ and let $\delta$ denote the Poincaré distance on the open unit disk $\Delta$ in the complex plane. If $D$ is convex, then the Kobayashi distance between two points $p, q \in D$ can be defined in terms of a single analytic disk joining $p$ and $q$ in $D:$ the function $d^{*}: D \times D \rightarrow \mathbf{R}$ defined by

$$
\begin{aligned}
d^{*}(p, q)=\inf _{f}\{\delta(a, b): f: \Delta \rightarrow D \text { holomorphic }, & \\
& f(a)=p, f(b)=q, a, b \in \Delta\}
\end{aligned}
$$

satisfies the triangle inequality, and $d^{*}$ is the Kobayashi distance function on $D[\mathbf{L 1}]$.

When $D$ is a pseudo-convex domain, $d^{*}$ does not in general satisfy the triangle inequality (see [L1] for example). To define the Kobayashi distance $d: D \times D \rightarrow \mathbf{R}$ on a pseudo-convex domain $D$, it is necessary to consider chains of analytic disks joining $p$ and $q$ :

$$
d(p, q)=\inf \left\{\delta\left(a_{1}, b_{1}\right)+\cdots+\delta\left(a_{n}, b_{n}\right): a_{i}, b_{j} \in \Delta\right\}
$$


where the infimum is taken over all possible chains of holomorphic functions $f_{k}: \Delta \rightarrow D, k=1, \ldots, n$, with length $n \geq 1$ joining $p$ and $q$, i.e.

$$
\begin{gathered}
f_{1}(0)=p, \quad f_{1}\left(b_{1}\right)=f_{2}\left(a_{2}, \ldots, f_{n-1}\left(b_{n-1}\right)=f_{n}\left(a_{n}\right),\right. \\
f_{n}\left(b_{n}\right)=q .
\end{gathered}
$$

Clearly, the two functions $d$ and $d^{*}$ satisfy the inequality $d \leq d^{*}$, and $d$ and $d^{*}$ coincide when the domain $D$ is convex. In particular, any two points $p, q$ in a convex domain can be joined by a single analytic disk and the Kobayashi distance between the two points can be realized by the Poincare distance on the disk. Although this is no longer true for pseudo-convex domains, one might hope that this property still holds if $p$ and $q$ are very close. We call a point $p \in D$ Kobayashi simple if $p$ has a neighborhood $U_{p}$ such that the Kobayashi distance between $p$ and any other point $q$ in $U_{p}$ can be realized by the Poincare distance on a single analytic disk joining $p$ and $q$. One of the main purposes of this paper is to examine the infinitesimal behavior of the Kobayashi distance function around a point $p$ in a pseudo-convex domain, and further, give obstructions for $p$ to be Kobayashi simple.

The infinitesimal behavior of the Kobayashi distance is closely related to the infinitesimal form of the Kobayashi distance introduced by Royden [R]. Let $T D$ denote the tangent space of $D$. We define the Kobayashi metric $F: T D \rightarrow \mathbf{R}$ as follows: For $v \in T_{p} D$, let $F(v)$ be the length of $v$ defined by

(1.4) $F(v)=\inf \left\{\frac{1}{\lambda_{f}}: f: \Delta \rightarrow D\right.$ holomorphic ,

$$
\left.f(0)=p, f^{\prime}(0)=\lambda_{f} v, \lambda_{f}>0\right\}
$$

where infimum is taken over $f$. We show that the length $F(v)$ of $v$ is in fact the directional derivative of the function $z \mapsto d^{*}(z, p)$ at $p$ in the direction of $v$ in the following sense:

3.18. Proposition. The following identity holds: For each $v \in T_{p} D$ with $\|v\|=1$,

$$
F(v)=\lim _{t \rightarrow 0} \frac{d^{*}(p+t v, p)}{|t|}
$$


We define the Kobayashi indicatrix $I_{p}$ to be the set $I_{p}=\{v \in$ $\left.T_{p} D: F(v)<1\right\}$. One of the main results of this paper is the following theorem.

THEOREM 4.1. If $p$ is Kobayashi simple, then $I_{p}$ is convex.

As a consequence of the theorem, if $I_{p}$ is not convex, then $p$ is not a Kobayashi simple point, and therefore there is a sequence of points $p_{n}$ convergent to $p$ such that the distance between $p_{n}$ and $p$ cannot be realized by the Poincaré distance on a single analytic disk joining $p_{n}$ and $p$.

Unlike the triangle inequality of $d^{*}$, convexity of the indicatrix of certain domains is relatively easy to check. It is well known that the Kobayashi indicatrix of a complete circular domain $D$ (i.e. a pseudoconvex domain such that $\lambda D \subset D$ for all $\lambda \in \Delta$ ) coincides with the domain $D$ itself under the natural identification of $T_{0} D$ with $\mathbf{C}^{m}$ [Ba]. By giving an example of non-convex complete circular domains (Example 4.22), we prove the following corollary:

4.13. Corollary. (1) If $D$ is a complete circular domain and $0 \in$ $D$ is Kobayashi simple, then $D$ is convex.

(2) There exist strongly pseudo-convex domains with a point that is not Kobayashi simple.

We call a domain Kobayashi simple if all of its points are Kobayashi simple. For example, all convex domains are Kobayashi simple (see $\S 2$ for a non-trivial example). According to Lempert's results in [L1], if $D$ is a strongly convex domain with $C^{k}$ boundary $(k \geq 6)$, then the Kobayashi metric of $D$ becomes a Finsler metric of class $C^{k-4}$ (i.e. the map $F: T D \rightarrow \mathbf{R}$ is $C^{k-4}$ away from the zero section and the indicatrix is strongly convex at any point in the domain (Definition 5.1)). We show that certain properties of strongly convex domains generalize to Kobayashi simple domains under the condition that the Kobayashi metric is a Finsler metric of class $C^{2}$. We call an analytic disk in $D$ joining a pair of points $p, q \in D$ extremal for $p, q$ if the distance $d^{*}(p, q)$ is realized by the Poincare distance on the disk (Definition 2.1). The properties of strongly convex domains which we consider here are the following:

THEOREM (Lempert). Let $D$ be a strongly convex domain with $C^{k}$ boundary $(k \geq 6)$. Then the following are true: 
(1) For any $p, q \in D$, there exists a unique extremal disk joining $p$ and $q$.

(2) Any geodesic curve of $F$ parametrized by arc length is (real) analytic.

For Kobayashi simple domains, we have the following results:

6.9. Corollary. Suppose that $D$ is Kobayashi simple and the Kobayashi metric $F$ of $D$ is a Finsler metric of class $C^{2}$. Then the following are true:

(1) Each point $p \in D$ has a neighborhood $U_{p}$ such that, for any $q \in U_{p}$, there is a unique extremal disk joining $p$ and $q$.

(2) Any geodesic curve of $F$ parametrized by arc length is (real) analytic.

6.11. Theorem. Let $D$ be a Kobayashi simple domain. Suppose that the Kobayashi metric $F$ is a complete Finsler metric of class $C^{2}$. Then any two points $p$ and $q$ in $D$ can be joined by a single analytic disk on which the Kobayashi distance $d(p, q)$ is realized by Poincaré distance on the analytic disk. In particular, the equality $d=d^{*}$ holds and the function $d^{*}$ satisfies the triangle inequality.

We remark that the work in this paper is motivated by the following conjecture by S. Krantz [Kr]:

Conjecture. Let $D$ be a strongly pseudo-convex domain. Then there is a fixed constant $K=K(D)$ such that the Kobayashi distance between any two points in $D$ can be realized by chain of holomorphic maps of length less than $K$.

If the domain $D$ satisfies the hypothesis of Theorem 6.11 , then the constant $K$ in the conjecture would be one. It would be interesting to see how far the hypothesis of Theorem 6.11 can be weakened. For general pseudo-convex domains, an interesting open question is whether the converse of Theorem 4.1 is true, and the Kobayashi simple points are characterized only by the convexity of the indicatrix.

Acknowledgment. I would like to express my thanks to S. Krantz for many helpful conversations and for the term "Kobayashi simple". I wish to thank T. Duchamp for conversations we had.

2. Definitions and basic facts on the Kobayashi metric. In this section, we introduce the notations and definitions, and review basic 
properties of the Kobayashi distance and the Kobayashi metric. For further properties, we refer the reader to $[\mathbf{K}]$ and $[\mathbf{R}]$.

Let $D_{1}$ and $D_{2}$ be bounded domains. We denote by $D_{2}\left(D_{1}\right)$ the set of all holomorphic functions from $D_{1}$ into $D_{2}$. We call $D$ taut if $D(\Delta)$ is a normal family. It is known that the concept of taut domain is closely related to the concept of pseudo-convex domain: taut domains are pseudo-convex [Wu]. Conversely, all the pseudoconvex domains with $C^{1}$ boundary are taut [Ker]. Note that, since $D$ is bounded, $D$ is a hyperbolic manifold in the sense of Kobayashi $[\mathbf{K}]$, and the Kobayashi distance $d$ induces the usual topology on $D$ [R]. If it is necessary to specify the domain on which $d$ and $F$ are defined, we use the subscripted notation $F_{D}$ and $d_{D}$ to denote the Kobayashi metric and the distance function of the domain $D$.

Although some of the results in this paper may generalize to a broader class of domains, we will restrict attention here to $D$ as bounded taut domain.

2.1. Definition. $A$ map $f \in D(\Delta)$ is called extremal for a pair of points $p, q \in D$ if $d^{*}(p, q)=\delta(a, b), f(a)=p$ and $f(b)=q$ for some $a, b \in \Delta$ (i.e. if $f$ attains the infimum in (1.1)). Similarly, $f \in D(\Delta)$ is called extremal for a tangent vector $v \in T_{p} D$ (or in the direction of $v$ ) if $f(0)=p$ and $f^{\prime}(0)=\frac{v}{F(v)}$ (i.e. if $\frac{1}{\lambda_{f}}$ attains the infimum in (1.4)). If $f: \Delta \rightarrow D$ is an extremal map, we call the image $f(\Delta)$ an extremal disk.

2.2. REMARK. We will often use the following facts in this paper:

(1) $A$ map $f \in D(\Delta)$ is extremal for $v \in T_{p} D$ if and only if the identity $F\left(f^{\prime}(0)\right)=1$ holds.

(2) If $f \in D(\Delta)$ is extremal for $p, q \in D$, we can find $b \in(0,1)$ such that $d^{*}(p, q)=\delta(0, b)$.

One of the advantages of considering taut domains is that the existence of extremal disks can be easily shown by applying the normal families argument:

2.3. Lemma. For any pair $p, q \in D$, there is at least one extremal map. Similarly, for any $v \in T_{p} D$, there is at least one extremal map.

The Kobayashi metric $F$, the Kobayashi distance $d$ and the function $d^{*}$ have the distance decreasing property under holomorphic 
maps: If $\phi \in D_{2}\left(D_{1}\right)$, then

(2.4)

$$
\begin{gathered}
d_{D_{1}}(p, q) \geq d_{D_{2}}(\phi(p), \phi(q)), \quad d_{D_{1}}^{*}(p, q) \geq d_{D_{2}}^{*}(\phi(p), \phi(q)) \\
\text { and } \quad F_{D_{1}}(v) \geq F_{D_{2}}\left(\phi_{*} v\right),
\end{gathered}
$$

for all $p, q \in D_{1}$ and $v \in T D_{1}$. In particular, if $D_{1} \subset D_{2}, F_{D_{1}} \leq F_{D_{2}}$ and $d_{D_{1}} \leq d_{D_{2}}$. If $\phi$ is a biholomorphism, equality holds in (2.4). This shows that $d, d^{*}$ and $F$ are invariant under biholomorphism.

On the open unit disk $\Delta$, the Kobayashi distance $d$ (and the function $d^{*}$ ) coincides with the Poincare distance $\delta$, and the Kobayashi metric $F$ coincides with the Poincaré metric $F_{\Delta}$ :

$$
F_{\Delta}(v)=\frac{|v|}{1-|z|^{2}}
$$

where $v \in T_{z} \Delta$. For $a, b \in(-1,1) \subset \Delta$ such that $a<b$, the Poincaré distance $\delta(a, b)$ is $\delta(a, b)=\tanh ^{-1} b-\tanh ^{-1} a$.

The Kobayashi distance $d$ can be equivalently defined in terms of $d^{*}$ as the largest distance dominated by $d^{*}$ :

$$
d(p, q)=\inf \left\{d^{*}\left(p, p_{1}\right)+\cdots+d^{*}\left(p_{n}, q\right)\right\}
$$

where the infimum is taken over all possible $p_{1}, \ldots, p_{n} \in D, n \geq$ 0 . If $D$ is a convex domain, Lempert showed that $d^{*}$ satisfies the triangle inequality and the identity $d^{*}=d$ holds [L1]. In other words, the distance between any two points $p, q$ in $D$ can be realized by $d^{*}(p, q)$. Here, we give a more precise definition of Kobayashi simple point in terms of $d^{*}$ :

2.7. Definition. A point $p \in D$ is called Kobayashi simple if $p$ has a neighborhood $U_{p} \subset D$ such that for any $q \in U_{p}$, the equality $d(p, q)=d^{*}(p, q)$ holds. A domain $D$ is called Kobayashi simple if all the points of $D$ are Kobayashi simple.

From Lempert's result, it is clear that all the convex domains are Kobayashi simple. Furthermore, products and holomorphic retracts of Kobayashi simple domains are again Kobayashi simple. Besides these examples, there are topologically non-trivial examples of Kobayashi simple domain. One example is

$$
D=\left\{(z, w) \in \mathbf{C}^{2}:(|z|-2)^{2}+|w|^{2}<1\right\} .
$$

In fact, this domain is a quotient space of a cylinder in $\mathbf{C}^{2}$ which is Kobayashi simple because of its convexity. More generally, one can 
obtain a class of Kobayashi simple domains by considering quotient spaces of Kobayashi simple domains in a similar manner.

We define a metric $\widetilde{F}: T D \rightarrow \mathbf{R}$ by replacing the unit disk $\Delta$ by the open unit ball $B$ in $\mathbf{C}^{m}$ in the definition of the Kobayashi metric $F$ in (1.4), and we note that this metric $\widetilde{F}$ coincides with the usual Kobayashi metric $F[\mathbf{R}]$.

Finally, we define the length of a curve in $D$ with respect to the Kobayashi metric $F$. If $\gamma:[a, b] \rightarrow D$ is a $C^{1}$ curve, the length $L(\gamma)$ is defined by the integral

$$
L(\gamma)=\int_{a}^{b} F\left(\gamma^{\prime}(t)\right) d t
$$

Note that the length $L(\gamma)$ does not change even if $\gamma$ is re-parametrized because of the homogeneity property of $F[\mathbf{R}]$ :

$$
F(a v)=|a| F(v), \quad \text { for } a \in \mathbf{C} \text { and } v \in T D .
$$

The Kobayashi distance $d$ is related to $F$ as follows [R]:

2.10. Theorem (Royden). Let $p, q$ be any points in $D$. The Kobayashi distance $d(p, q)$ is the integrated distance, i.e.

$$
d(p, q)=\inf _{\gamma} L(\gamma)
$$

where the infimum is taken over all possible $C^{1}$ curves in $D$ joining $p$ and $q$.

3. Infinitesimal behavior of the Kobayashi distance. In this section, we study the relation between the infinitesimal behavior of the Kobayashi distance and the Kobayashi metric $F$. First, we begin by stating some of Lempert's results on convex domains.

3.1. TheOREM (Lempert). Suppose $D$ is a strongly convex domain with smooth boundary. Then the following statements are true:

(1) For each tangent vector $v \in T D$ (resp. for each pair of points $p, q \in D$ ), there is a unique extremal map corresponding to $v$ (resp. for $p, q)$.

(2) Extremal maps are proper imbeddings that smoothly extend to the closed disk $\bar{\Delta}$.

(3) Extremal maps are isometries with respect to the distances $\delta$ and $d$, and the metrics $F_{\Delta}$ and $F$. Consequently, if $f: \Delta \rightarrow D$ is an extremal map (either for a tangent vector or for a pair of points $p, q$ in $D)$, then the disk $f(\Delta)$ is the extremal disk for any vector tangent to $f(\Delta)$ and for any pair of points on $f(\Delta)$. 
The following proposition is well known.

3.2. Proposition [R]. For any taut domain $D$, the Kobayashi metric $F: T D \rightarrow \mathbf{R}$ is continuous.

3.3. Lemma. Let $D$ be a strongly convex domain with smooth boundary. Suppose that $\left\{p_{n}\right\}$ and $\left\{q_{n}\right\}$ are sequences in $D$ and $\left\{t_{n}\right\}$ is a sequence in $(0,1)$ such that

$$
\lim _{n \rightarrow \infty} p_{n}=\lim _{n \rightarrow \infty} q_{n}=p, \quad \text { and } \quad \lim _{n \rightarrow \infty} \frac{q_{n}-p_{n}}{t_{n}}=v \neq 0 .
$$

Then the extremal maps $f_{n}: \Delta \rightarrow D$ for $p_{n}, q_{n}$ converge to the unique extremal map $f: \Delta \rightarrow D$ for $v \in T_{p} D$.

Proof. By the extremality of $f_{n}$, we can find $b_{n} \in(0,1)$ such that $f_{n}(0)=p_{n}, f_{n}\left(b_{n}\right)=q_{n}$ and $\delta\left(0, b_{n}\right)=d^{*}\left(p_{n}, q_{n}\right)$. Clearly $b_{n} \rightarrow 0$ as $n \rightarrow \infty$.

Let $f_{n_{k}}$ be any subsequence of $f_{n}$ which converges to a holomorphic map $f$ in $D(\Delta)$ uniformly on compact sets. Note that strong convexity of $D$ implies that $D$ is taut, and hence, $f_{n}$ has at least one such subsequence [Gr]. By Theorem 3.1, $f_{n}$ is extremal in the direction of $f_{n}^{\prime}(0)$, and hence $F\left(f_{n}^{\prime}(0)\right)=1$. This implies that $f$ is also extremal in the direction of $f^{\prime}(0)$ since

$$
F\left(f^{\prime}(0)\right)=F\left(\lim _{k \rightarrow \infty} f_{n_{k}}^{\prime}(0)\right)=\lim _{k \rightarrow \infty} F\left(f_{n_{k}}^{\prime}(0)\right)=1 .
$$

On the other hand, note that the following identity holds:

$$
\lim _{n \rightarrow \infty} \frac{q_{n_{k}}-p_{n_{k}}}{b_{n_{k}}}=\lim _{n \rightarrow \infty} \frac{f_{n_{k}}\left(b_{n_{k}}\right)-f_{n_{k}}(0)}{b_{n_{k}}}=f^{\prime}(0) .
$$

Combining this with the last identity in (3.4), we can easily see that $f^{\prime}(0)$ and $v$ are parallel, and hence, $f$ is extremal for $v$. By the uniqueness of the extremal map for $v$, any convergent subsequence of $f_{n}$ must converge to the same $f$, and hence it follows that $f_{n}$ converges to $f$ uniformly on compact sets.

We show that Lemma 3.3 generalizes to the case when $D$ is any taut domain:

3.6. Theorem. Let $\left\{p_{n}\right\}$ and $\left\{q_{n}\right\}$ be sequences in $D$ both convergent to $p \in D$. Suppose that $f_{n}$ are extremal maps for the pairs $p_{n}, q_{n}$ and that they converge to $f: \Delta \rightarrow D$ uniformly on compact 
sets. Then, $f^{\prime}(0) \neq 0$ and $f$ is an extremal map in the direction of $f^{\prime}(0) \in T_{p} D$. Moreover, the following identity holds:

$$
\lim _{n \rightarrow \infty} \frac{d^{*}\left(p_{n}, q_{n}\right)}{\left\|p_{n}-q_{n}\right\|}=\frac{F(v)}{\|v\|}
$$

where $v \in T_{p} D$ is any non-zero vector parallel to $f^{\prime}(0)$ and \|\| denotes the Euclidean norm on $T_{p} D \cong \mathrm{C}^{m}$.

Proof. By the extremality of $f_{n}$ again, we can find $t_{n} \in(0,1)$ such that $f_{n}(0)=p_{n}, f_{n}\left(t_{n}\right)=q_{n}$ and $\delta\left(0, t_{n}\right)=d^{*}\left(p_{n}, q_{n}\right)$. Clearly $t_{n} \rightarrow 0$ as $n \rightarrow \infty$.

First we will prove that $f^{\prime}(0)$ is non-zero. Choose an open Euclidean ball $B(p, r) \subset D$ of small radius $r$ centered at $p$. Then, by the compactness of the closed ball $\bar{B}(p, r / 2)$, we can find a constant $C$ such that $d_{B(p, r)}\left(z_{1}, z_{2}\right) \leq C\left\|z_{1}-z_{2}\right\|$ for $z_{1}, z_{2} \in \bar{B}(p, r / 2)$. Recall the inequality $d_{D}^{*} \leq d_{B(p, r)}^{*}$. Hence, for large $n$, we have the inequalities

$$
\begin{aligned}
\frac{\delta\left(0, t_{n}\right)}{t_{n}} & =\frac{d_{D}^{*}\left(f_{n}\left(t_{n}\right), f_{n}(0)\right)}{t_{n}} \\
& \leq \frac{d_{B(p, r)}\left(f_{n}\left(t_{n}\right), f_{n}(0)\right)}{t_{n}} \leq C\left\|\frac{f_{n}\left(t_{n}\right)-f_{n}(0)}{t_{n}}\right\| .
\end{aligned}
$$

This implies that $f^{\prime}(0)$ is non-zero since the first term converges to 1 while the last term converges to $f^{\prime}(0)$.

To show that $f$ is extremal, recall that the metric $\widetilde{F}$ coincides with the usual Kobayashi metric $F$. Therefore, for each $\epsilon>0$, there is a holomorphic map $\varphi: B \rightarrow D$ such that

$$
F\left(f^{\prime}(0)\right) \leq \frac{1}{\lambda_{\varphi}}<F\left(f^{\prime}(0)\right)+\epsilon,
$$

$\varphi(0)=p, \varphi_{*}\left(\frac{\partial}{\partial x^{1}}\right)=\lambda_{\varphi} f^{\prime}(0)$ and $\operatorname{det}\left(\varphi_{*}\right)_{0} \neq 0$. By the inverse function theorem, we can find a neighborhood $U$ of $0 \in B$ such that the restriction map $\left.\varphi\right|_{U}$ is a biholomorphism from $U$ to an open neighborhood $V$ of $p \in D$. Without loss of generality, we may assume $p_{n}, q_{n} \in V$, and since $\left.\varphi\right|_{U}$ is a biholomorphism between $U$ and $V$, there are sequences $\tilde{p}_{n}, \tilde{q}_{n} \in U$ such that $\varphi\left(\tilde{p}_{n}\right)=p_{n}$ and $\varphi\left(\tilde{q}_{n}\right)=q_{n}$. For each $n$, we can take an (unique) extremal map $h_{n}: \Delta \rightarrow B$ into the ball for $\tilde{p}_{n}$ and $\tilde{q}_{n}$ such that $\tilde{p}_{n}=h_{n}(0)$ and $\tilde{q}_{n}=h_{n}\left(s_{n}\right)$ for some $s_{n} \in(0,1)$. Note that the sequences $\tilde{q}_{n}, \tilde{p}_{n}$ and $t_{n}$ satisfy all the conditions in (3.4) since both sequences $\tilde{q}_{n}$ and 
$\tilde{p}_{n}$ converges to 0 in $B$, and

$$
\text { (3.10) } \begin{aligned}
\frac{\tilde{q}_{n}-\tilde{p}_{n}}{t_{n}} & =\frac{\varphi^{-1}\left(q_{n}\right)-\varphi^{-1}\left(p_{n}\right)}{t_{n}} \\
& =\frac{\varphi^{-1}\left(f_{n}\left(t_{n}\right)\right)-\varphi^{-1}\left(f_{n}(0)\right)}{t_{n}} \longrightarrow\left(\varphi^{-1} \circ f\right)^{\prime}(0) \neq 0
\end{aligned}
$$

as $n \rightarrow \infty$. Thus, by Lemma 3.3 , the sequence $\left\{h_{n}\right\}$ converges to the unique extremal map $h: \Delta \rightarrow B$.

Note that each $n$, both the maps $\varphi \circ h_{n}: \Delta \rightarrow D$ and $f_{n}$ are joining $p_{n}$ and $q_{n}$, i.e.

$$
\begin{aligned}
& \varphi \circ h_{n}(0)=\varphi\left(\tilde{p}_{n}\right)=p_{n}=f_{n}(0), \\
& \varphi \circ h_{n}\left(s_{n}\right)=\varphi\left(\tilde{q}_{n}\right)=q_{n}=f_{n}\left(t_{n}\right) .
\end{aligned}
$$

Recall that the $f_{n}$ are extremals for $p_{n}$ and $q_{n}$. Hence

(3.12) $\delta\left(0, t_{n}\right)=d^{*}\left(p_{n}, q_{n}\right)=d^{*}\left(\varphi \circ h_{n}(0), \varphi \circ h_{n}\left(s_{n}\right)\right) \leq \delta\left(0, s_{n}\right)$.

This implies $t_{n} \leq s_{n}$, and hence $1 \leq \frac{s_{n}}{t_{n}}$.

Since $f_{n}$ converges to $f$ uniformly on compact sets, we have

$$
\begin{aligned}
f^{\prime}(0) & =\lim _{n \rightarrow \infty} \frac{f_{n}\left(t_{n}\right)-f_{n}(0)}{t_{n}}=\lim _{n \rightarrow \infty} \frac{q_{n}-p_{n}}{t_{n}} \\
& =\lim _{n \rightarrow \infty}\left\{\frac{\varphi \circ h_{n}\left(s_{n}\right)-\varphi \circ h_{n}(0)}{s_{n}} \frac{s_{n}}{t_{n}}\right\} .
\end{aligned}
$$

But since we have

$$
\lim _{n \rightarrow \infty}\left\{\frac{\varphi \circ h_{n}\left(s_{n}\right)-\varphi \circ h_{n}(0)}{s_{n}}\right\}=(\varphi \circ h(0))^{\prime} \neq 0
$$

the sequence $\frac{s_{n}}{t_{n}} \geq 1$ converges to some number $A \geq 1$. Therefore, from (3.13) and (3.14), we obtain

$$
f^{\prime}(0)=A(\varphi \circ h(0))^{\prime}=A \varphi_{*}\left(h^{\prime}(0)\right) .
$$

On the other hand, recall that $\varphi_{*}\left(\frac{\partial}{\partial x^{1}}\right)=\lambda_{\varphi} f^{\prime}(0)$. Combining this with the identity (3.15), we have

$$
A h^{\prime}(0)=\frac{1}{\lambda_{\varphi}} \frac{\partial}{\partial x^{1}} \text {. }
$$

By applying the Kobayashi metric $F_{B}$ of the ball on both sides of this identity, and using the fact that $h$ is an extremal map (i.e. $F_{B}\left(h^{\prime}(0)\right)=$ 1), we obtain the identity $A \lambda_{\varphi}=1$. Now, by the inequality (3.9), we have

$$
1 \leq A=\frac{1}{\lambda_{\varphi}}<F\left(f^{\prime}(0)\right)+\epsilon
$$


Recall that the inequality $F\left(g^{\prime}(0)\right) \leq 1$ holds for any $g \in D(\Delta)$. In particular, $F\left(f^{\prime}(0)\right) \leq 1$, and since $\epsilon>0$ in the inequality (3.17) was arbitrarily chosen, we conclude that $F\left(f^{\prime}(0)\right)=1$. This proves that $f$ is extremal in the direction of $f^{\prime}(0)$.

To prove the identity (3.7), recall from (3.13) that

$$
f^{\prime}(0)=\lim _{n \rightarrow \infty} \frac{q_{n}-p_{n}}{t_{n}}
$$

Also, recall that $\delta\left(0, t_{n}\right)=d^{*}\left(p_{n}, q_{n}\right)$ and $\lim _{n \rightarrow \infty} \frac{\delta\left(0, t_{n}\right)}{t_{n}}=1$. Using these identities and the fact that $f$ is extremal (i.e. ${ }^{n} F\left(f^{\prime}(0)\right)=1$ ), the identity (3.7) can be verified as follows

$$
\lim _{n \rightarrow \infty} \frac{d^{*}\left(p_{n}, q_{n}\right)}{\left\|p_{n}-q_{n}\right\|}=\lim _{n \rightarrow \infty} \frac{\delta\left(0, t_{n}\right) / t_{n}}{\left\|\frac{p_{n}-q_{n}}{t_{n}}\right\|}=\frac{1}{\left\|f^{\prime}(0)\right\|}=\frac{F\left(f^{\prime}(0)\right)}{\left\|f^{\prime}(0)\right\|}
$$

3.18. Proposition. The following identity holds: For each $v \in T_{p} D$ with $\|v\|=1$,

$$
F(v)=\lim _{t \rightarrow 0} \frac{d^{*}(p+t v, p)}{|t|}
$$

Proof. We prove this using Theorem 3.6. Let $s_{n} \in \mathbf{R}$ be a sequence convergent to 0 . Also, let, for each $n, f_{n} \in D(\Delta)$ be an extremal map for the pair of points $p_{n}=p, q_{n}=p+s_{n} v$ such that $p_{n}=f_{n}(0)$ and $q_{n}=f_{n}\left(t_{n}\right)$ for some sequence $t_{n} \in(0,1)$ that converges to 0 . By taking subsequence if necessary, we may assume that $f_{n}$ converges to $f \in D(\Delta)$ uniformly on compact sets. To apply Theorem 3.6, we verify that $f^{\prime}(0)$ is parallel to $v$ :

$$
f^{\prime}(0)=\lim _{n \rightarrow \infty} \frac{f_{n}\left(t_{n}\right)-f_{n}(0)}{t_{n}}=\lim _{n \rightarrow \infty} \frac{\left(p+s_{n} v\right)-p}{t_{n}}=\lim _{n \rightarrow \infty} \frac{s_{n}}{t_{n}} v .
$$

We can then apply Theorem 3.6 to obtain the following identity:

$$
F(v)=\lim _{n \rightarrow \infty} \frac{d^{*}\left(p, p+s_{n} v\right)}{\left\|p-\left(p-s_{n} v\right)\right\|}=\lim _{n \rightarrow \infty} \frac{d^{*}\left(p, p+s_{n} v\right)}{\left|s_{n}\right|}
$$

Since $s_{n}$ was arbitrary, the identity (3.19) follows.

4. Domains with local properties of convex domains. In this section, we prove Theorem 4.1 in the introduction. 
4.1. Theorem. If $p \in D$ is Kobayashi simple, then $I_{p}$ is convex.

Proof. Suppose that $I_{p}$ is not convex for some $p \in D$. Without loss of generality, we may assume that $p$ is the origin $0 \in \mathbf{C}^{m}$. We will show that there are sequences $p_{n}$ and $q_{n}$ in $D$ both convergent to 0 such that

$$
d^{*}\left(0, q_{n}\right)+d^{*}\left(q_{n}, p_{n}\right)<d^{*}\left(0, p_{n}\right) .
$$

This inequality then shows that $p$ is not a Kobayashi simple point because

$$
d\left(0, p_{n}\right) \leq d^{*}\left(0, q_{n}\right)+d^{*}\left(q_{n}, p_{n}\right)<d^{*}\left(0, p_{n}\right) .
$$

Since we assumed that $I_{0}$ is not convex, there are two vectors $u, v \in T_{0} D$, such that

$$
F(u)+F(v)<F(u+v) .
$$

Let $w=u+v$ and $f_{u}, f_{v}, f_{w}: \Delta \rightarrow D$ be extremal maps in the directions of $u, v$ and $w$ such that $f_{u}(0)=f_{v}(0)=f_{w}(0)=0$. Note that, since the curves $\gamma_{u}(t)=f_{u}(t)$ and $\gamma_{w}(t)=f_{w}(t), t \in(-1,1)$ are tangent to $u, w$ at $0 \in D$ and $v \in \operatorname{span}\{u, w\}$, we can find sequences $a_{n}, b_{n} \in(0,1)$ convergent to 0 such that, if we denote

$$
p_{n}=f_{u}\left(a_{n}\right), \quad q_{n}=f_{w}\left(b_{n}\right),
$$

then

$$
\lim _{n \rightarrow \infty} \frac{q_{n}-p_{n}}{\left\|q_{n}-p_{n}\right\|}=\frac{v}{\|v\|} .
$$

Let $f_{n}: \Delta \rightarrow D$ be extremal maps for $p_{n}$ and $q_{n}$. Clearly, we can take $f_{n}$ such that $p_{n}=f_{n}(0)$ and $q_{n}=f_{n}\left(c_{n}\right)$ for some $c_{n} \in(0,1)$. By a normal families argument, and by taking a subsequence of $f_{n}$ if necessary, we may assume that $f_{n}$ converges to a function $f \in D(\Delta)$ uniformly on compact sets. Hence

$$
\lim _{n \rightarrow \infty} \frac{q_{n}-p_{n}}{\left\|q_{n}-p_{n}\right\|}=\lim _{n \rightarrow \infty} \frac{\left(f_{n}\left(c_{n}\right)-f_{n}(0)\right) / c_{n}}{\left\|\frac{f_{n}\left(c_{n}\right)-f_{n}(0)}{c_{n}}\right\|}=\frac{f^{\prime}(0)}{\left\|f^{\prime}(0)\right\|}
$$

Comparing this with the identity (3.7), we obtain $\frac{v}{\|v\|}=\frac{f^{\prime}(0)}{\left\|f^{\prime}(0)\right\|}$. Moreover, by Theorem 3.6, the map $f$ is an extremal in the direction of $f^{\prime}(0)$. Therefore,

$$
f^{\prime}(0)=\frac{v}{F(v)}
$$


The basic idea of the proof is to approximate the elements of the inequality (4.4) with the sequences $d^{*}\left(0, p_{n}\right), d^{*}\left(0, q_{n}\right)$ and $d^{*}\left(q_{n}, p_{n}\right)$. By taking subsequences if necessary, we may again assume that the extremal maps joining the pairs $0, p_{n}$ and $0, q_{n}$ are convergent. Hence we may apply Theorem 3.6 to these pairs of points. Then the identity (3.7) implies

$$
\begin{aligned}
& \lim _{n \rightarrow \infty} \frac{d^{*}\left(0, p_{n}\right)}{\left\|p_{n}\right\|}\|u\|=F(u), \\
& \lim _{n \rightarrow \infty} \frac{d^{*}\left(0, q_{n}\right)}{\left\|q_{n}\right\|}\|w\|=F(w)
\end{aligned}
$$

as $n \rightarrow \infty$. We obtain a similar identity for the pair $p_{n}$ and $q_{n}$ as follows. Recall that $f_{n}$ is extremal, and hence $d^{*}\left(p_{n}, q_{n}\right)=\delta\left(0, c_{n}\right)$.

By the identities (4.5) and (4.8) and the fact that $\delta\left(0, c_{n}\right) / c_{n} \rightarrow 1$ as $n \rightarrow \infty$, we obtain the identity

$$
\begin{aligned}
\lim _{n \rightarrow \infty} \frac{d^{*}\left(q_{n}, p_{n}\right)}{\left\|q_{n}-p_{n}\right\|}\|v\| & =\lim _{n \rightarrow \infty} \frac{\frac{\delta\left(0, c_{n}\right)}{c_{n}}}{\left\|\frac{f_{n}\left(c_{n}\right)-f_{n}(0)}{c_{n}}\right\| v \|} \\
& =\frac{\|v\|}{\left\|f^{\prime}(0)\right\|}=F(v) .
\end{aligned}
$$

Now we consider the inequality (4.4). Since the inequality is strict, we can find numbers $c$ and a small $\epsilon>0$ such that

$$
F(u)+F(v)<c<c+\epsilon<F(w) .
$$

From the identities (4.9)-(4.11), we conclude that

$$
\frac{d^{*}\left(0, p_{n}\right)}{\left\|p_{n}\right\|}\|u\|+\frac{d^{*}\left(q_{n}, p_{n}\right)}{\left\|q_{n}-p_{n}\right\|}\|v\|<c<c+\epsilon<\frac{d^{*}\left(0, q_{n}\right)}{\left\|q_{n}\right\|}\|w\|
$$

for $n$ large. If we let

$$
\begin{gathered}
M_{n}=\frac{\|u\|}{\left\|p_{n}\right\|} \frac{\left\|q_{n}\right\|}{\|w\|}, \quad N_{n}=\frac{\|v\|}{\left\|q_{n}-p_{n}\right\|} \frac{\left\|q_{n}\right\|}{\|w\|}, \\
R_{n}=d^{*}\left(0, q_{n}\right) \frac{\|w\|}{\left\|q_{n}\right\|}
\end{gathered}
$$

then the inequality (4.13) can be written as

$$
\frac{d^{*}\left(0, p_{n}\right) M_{n}+d^{*}\left(q_{n}, p_{n}\right) N_{n}}{d^{*}\left(0, q_{n}\right)}<c R_{n}^{-1}<(c+\epsilon) R_{n}^{-1}<1
$$

Note that, from the inequality (4.13) again, we have $c+\epsilon<R_{n}$, and this implies

$$
c R_{n}^{-1}<\frac{c}{c+\epsilon}<1
$$


To complete the proof, we claim that $M_{n}, N_{n} \rightarrow 1$. Once the claim is proved, we have

$$
\frac{c}{c+\epsilon}<M_{n}, \quad \frac{c}{c+\epsilon}<N_{n},
$$

for large $n$ and the inequality (4.15) together with (4.16) implies the following inequality:

$$
\frac{c}{c+\epsilon}\left(\frac{d^{*}\left(0, p_{n}\right)+d^{*}\left(q_{n}, p_{n}\right)}{d^{*}\left(0, q_{n}\right)}\right)<c R_{n}^{-1}<\frac{c}{c+\epsilon},
$$

for large $n$. The inequality (4.2) is a trivial consequence of this inequality. This completes the proof of the theorem.

To prove the claim, combine the identity (4.7) and (4.8) to obtain

$$
\lim _{n \rightarrow \infty} \frac{q_{n}-p_{n}}{\left\|q_{n}-p_{n}\right\|} \longrightarrow \frac{v}{\|v\|}=\frac{w}{\|v\|}-\frac{u}{\|v\|} .
$$

On the other hand,

$$
\frac{q_{n}-p_{n}}{\left\|q_{n}-p_{n}\right\|}=\frac{q_{n}}{\left\|q_{n}\right\|} \frac{\left\|q_{n}\right\|}{\left\|q_{n}-p_{n}\right\|}-\frac{p_{n}}{\left\|p_{n}\right\|} \frac{\left\|p_{n}\right\|}{\left\|q_{n}-p_{n}\right\|} .
$$

Note that we have the following identities:

(4.19) $\lim _{n \rightarrow \infty} \frac{q_{n}}{\left\|q_{n}\right\|}=\lim _{n \rightarrow \infty} \frac{f_{w}\left(b_{n}\right)-f_{w}(0)}{\left\|f_{w}\left(b_{n}\right)-f_{w}(0)\right\|}=\frac{f_{w}^{\prime}(0)}{\left\|f_{w}^{\prime}(0)\right\|}=\frac{w}{\|w\|}$,

(4.20) $\lim _{n \rightarrow \infty} \frac{p_{n}}{\left\|p_{n}\right\|}=\lim _{n \rightarrow \infty} \frac{f_{u}\left(a_{n}\right)-f_{u}(0)}{\left\|f_{u}\left(a_{n}\right)-f_{u}(0)\right\|}=\frac{f_{u}^{\prime}(0)}{\left\|f_{u}^{\prime}(0)\right\|}=\frac{u}{\|u\|}$.

Comparison of (4.17) and (4.18) together with (4.19) and (4.20) yields $M_{n}, N_{n} \rightarrow 1$.

We now conclude this section by giving an example of a family of domains that are not Kobayashi simple.

4.21. THEOREM [Ba]. If $D$ is complete circular, then the Kobayashi indicatrix $I_{0}$ at $0 \in D$ coincides with the domain $D$ under the natural identification of $T_{0} D$ with $D$.

There are many examples of non-convex complete circular pseudoconvex domains. We give one of them here:

4.22. Example. Consider the domain defined by

$$
D=\left\{(z, w) \in \mathbf{C}^{2}:|z|^{2}+|w|^{2}+t|z w|^{2}<1\right\} .
$$

This domain is clearly complete circular, and for large $t>0, D$ is non-convex. Also,note that $D$ is a strongly pseudo-convex domain 
with (real) analytic boundary. By Theorem 4.21 and Theorem 4.1, the indicatrix $I_{0}$ is non-convex, and 0 is not a Kobayashi simple point.

The following corollary is an easy consequence of Theorem 4.1, Theorem 4.21 and Example 4.22:

4.23. Corollary. (1) If $D$ is a complete circular domain and $0 \in$ $D$ is Kobayashi simple, then $D$ is convex.

(2) Not all pseudo-convex domains are Kobayashi simple.

5. The complex Finsler metrics. In this section, we briefly review the theory of Finsler metrics necessary for the next section. For more details about the theory of Finsler metric and the calculus of variations, we refer the reader to $[\mathbf{M M}]$ and $[\mathbf{S}]$.

5.1. Definition. A function $F: T D \rightarrow \mathbf{R}$ is called a Finsler metric of class $C^{2}$ if the following conditions are satisfied:

(1) $F$ is $C^{2}$ on the tangent space $T D$ away from the zero section.

(2) The indicatrix $I_{p}=\left\{v \in T_{p} D: F(v)<1\right\}$ is strongly convex for each $p \in D$.

(3) (Positive) homogeneity: $F(a v)=|a| F(v)$ for all $a \in \mathbf{R}$ and $v \in T D$.

If the homogeneity condition (3) holds for $a \in \mathbf{C}$, then we call $F$ a complex Finsler metric.

5.2. Remark. The condition on the indicatrix in condition (2) is in fact equivalent to the condition that the Hessian $\frac{\partial^{2} F^{2}}{\partial u^{i} \partial u^{j}}(v)$ at $v$ is positive definite for all $v \neq 0$ in $T D$, where $\left(u^{1}, \ldots, u^{2 n}\right)$ are any linear coordinate functions on a fiber of $T D$. Note that strong convexity implies convexity, which is equivalent to the condition that $F$ satisfies the triangle inequality

$$
F\left(v_{1}+v_{2}\right) \leq F\left(v_{1}\right)+F\left(v_{2}\right) .
$$

An easy example of a complex Finsler metric is the norm $\|v\|=$ $\sqrt{g(v, v)}$ induced by a Hermitian metric $g$ on $D$ if we regard it as a function defined on $T D$. Also note that, from Lempert's results, the Kobayashi metric on strongly convex domains with smooth boundary gives another important class of complex Finsler metrics [L1] [L2].

If $D$ is equipped with a Finsler metric $F$, we define the length $L(\gamma)$ of a curve $\gamma:[a, b] \rightarrow D$ by the formula (2.9). Note that the 
Finsler metric $F$ induces a distance function $d_{F}$ on $D$ in an obvious way by formula (2.11).

A $C^{1}$ curve $\gamma:(a, b) \rightarrow D$ is called a geodesic if it is a locally length minimizing curve i.e. if for any $t \in(a, b)$ there is neighborhood $(t-\epsilon, t+\epsilon) \subset(a, b)$ of $t$ such that the distance $d_{F}(\gamma(t-\epsilon), \gamma(t+\epsilon))$ is precisely the length $\int_{t-\epsilon}^{t+\epsilon} F\left(\gamma^{\prime}(t)\right) d t$ of the curve $\gamma_{\mid(t-\epsilon, t+\epsilon)}$.

It is not clear whether the Kobayashi metric $F$ has geodesics. However, for a Finsler metric $F$, the usual technique of the theory of calculus of variations applies, and the geodesics can be described as a solution of an ordinary differential equation (the Euler-Lagrange equation) [GF] [S]. Thus, for each tangent vector $v \in T_{p} D$, there is a unique geodesic $\gamma_{v}:(-\epsilon, \epsilon) \rightarrow D$ (in the sense that it is a unique solution of an ordinary differential equation) such that $\gamma(0)=p$ and $\gamma^{\prime}(0)=v$. It is known that for each $p \in D$, there there is a neighborhood $V$ of 0 in $T_{p} D$ such that, for any vector $v$ in $V$, the curve $\gamma_{v}$ is defined at least on the interval $[0,1]$. As in Hermitian geometry, we can define an exponential map exp: $V \rightarrow D$ at $p$ by $\exp (v)=\gamma_{v}(1)$.

5.4. TheOREM [MM]. Suppose $D$ is equipped with a Finsler metric. Then for each point $p \in D$, there is a neighborhood $V$ of 0 in $T_{p} D$ such that the exponential map exp: $V \rightarrow D$ is a $C^{1}$ diffeomorphism from $V$ onto an open set in $D$.

5.5. THEOREM (Whitehead). Suppose $D$ is equipped with a Finsler metric. Then for each point $p \in D$, there is a neighborhood $U_{p}$ of $p$ such that any two points in $U_{p}$ can be joined by a unique geodesic segment lying in $U_{p}$.

5.6. Definition. A Finsler metric $F$ defined on $D$ is geodesically complete if any geodesic segment $\gamma:(a, b) \rightarrow D$ (parametrized by its arc length) can be extended to a geodesic in $D$ defined on $\mathbf{R}$.

Note that if $F$ is geodesically complete on $D$, then the exponential map at $p$ is defined on $T_{p} D$.

5.7. THEOREM [MM]. (1) The metric $d_{F}$ induces the usual topology on $D$.

(2) A Finsler metric $F$ on $D$ is geodesically complete if and onty if the induced distance $d_{F}$ is complete as a topological metric.

(3) If $F$ is geodesically complete, then any two points $p, q$ in $D$ can be joined by a geodesic curve $\gamma$ with length $L(\gamma)=d_{F}(p, q)$ (i.e. $\gamma$ is the shortest path joining $p$ and $q$ ). 
6. Kobayashi simple domains and geodesics. In this section, we prove Corollary 6.9 and Theorem 6.11 in the introduction. An interesting property of a Kobayashi simple domain is that, if $p$ and $q$ are close enough, then there is a (real) analytic geodesic joining $p$ and $q$. Throughout this section, $x+i y$ denotes the standard euclidean coordinate on $\mathbf{C}$.

6.1. Lemma. Suppose that $p, q$ are points in $D$ satisfying the condition $d^{*}(p, q)=d(p, q)$. Let $f: \Delta \rightarrow D$ be an extremal map for $p, q$ such that $f(a)=p$ and $f(b)=q$ for some $a \in[0,1)$ and $b \in(a, 1)$. Then the following are true:

(1) $f_{*}$ is an isometry between $F$ and $F_{\Delta}$ along $[a, b]$ (i.e. we have $f^{*} F=F_{\Delta}$ at each $\left.x \in[a, b]\right)$. Consequently, the length of the curve $\gamma:[a, b] \rightarrow D$ defined by $\gamma(t)=f(t)$ is $\delta(a, b)=d^{*}(p, q)=d(p, q)$.

(2) The curve $\gamma$ is the shortest curve joining $p$ and $q$. Since $f$ is analytic, $\gamma(t)$ is a (real) analytic geodesic.

(3) $f$ is an extremal map joining $p$ and $f(x)$ for each $x \in(a, b]$. Also, the identity $d^{*}(p, f(x))=d(p, f(x))$ holds for all $x \in[a, b]$.

Proof. We give the proof in the order (1), (2), (3).

(i) To prove (1), observe that, by the length decreasing property of the Kobayashi metric,

$$
F\left(\gamma^{\prime}(t)\right)=F\left(\frac{\partial f}{\partial x}(t)\right)=F\left(\left.f_{*} \frac{\partial}{\partial x}\right|_{x=t}\right) \leq F_{\Delta}\left(\left.\frac{\partial}{\partial x}\right|_{x=t}\right) .
$$

By Royden's theorem, and the extremality of $f\left(\right.$ i.e. $d^{*}(p, q)=$ $\delta(a, b))$, we have

$$
\begin{aligned}
d(p, q) & \leq \int_{a}^{b} F\left(\gamma^{\prime}(t)\right) d t \leq \int_{a}^{b} F_{\Delta}\left(\left.\frac{\partial}{\partial x}\right|_{x=t}\right) d t \\
& =\delta(a, b)=d^{*}(p, q)=d(p, q) .
\end{aligned}
$$

From this, we obtain the identity

$$
\int_{a}^{b}\left\{F_{\Delta}\left(\left.\frac{\partial}{\partial x}\right|_{x=t}\right)-F\left(\gamma^{\prime}(t)\right)\right\} d t=0
$$

Since the function in the integral is non-negative, it has to vanish, and this implies that $f$ is an isometry.

(ii) To prove that $\gamma$ is a geodesic, we show that $\gamma$ is the shortest path joining $p$ and $q$. Suppose that $\sigma:[0, c] \rightarrow D$ is another curve 
joining $p, q$. From the inequality (6.3) and Royden's theorem again, we compare the length of $\gamma$ and $\sigma$ as follows:

$$
L(\gamma)=\int_{a}^{b} F\left(\gamma^{\prime}(t)\right) d t=d(p, q) \leq \int_{0}^{c} F\left(\sigma^{\prime}(t)\right) d t=L(\sigma) .
$$

(iii) To verify the statements (3), we claim that the inequality

$$
\int_{a}^{x} F\left(\gamma^{\prime}(t)\right) d t \leq d(p, \gamma(x))
$$

holds: From this inequality and the fact that $f$ is an isometry along $[a, b]$ (part (1)), we obtain the following inequality:

$$
\begin{aligned}
d^{*}(p, \gamma(x)) & \leq \delta(a, x)=\int_{a}^{x} F_{\Delta}\left(\left.\frac{\partial}{\partial x}\right|_{x=t}\right) d t \\
& =\int_{a}^{x} F\left(\gamma^{\prime}(t)\right) d t \leq d(p, \gamma(x)) \leq d^{*}(p, \gamma(x)) .
\end{aligned}
$$

This implies the equality $d^{*}(p, \gamma(x))=d(p, \gamma(x))=\delta(a, x)$. The extremality of $f$ follows since $d^{*}(p, \gamma(x))=\delta(a, x)$.

To prove the claim, suppose that the inequality (6.4) fails to hold i.e.

$$
d(p, \gamma(x))<\int_{a}^{x} F\left(\gamma^{\prime}(t)\right) d t
$$

Note that, by Royden's theorem again, we also have $d(\gamma(x), q) \leq$ $\int_{x}^{b} F\left(\gamma^{\prime}(t)\right) d t$. Combining these two inequalities, and by (6.3) again, we reach a contradiction:

$$
\begin{aligned}
d(p, q) & \leq d(p, \gamma(x))+d(\gamma(x), q)<\int_{a}^{x} F\left(\gamma^{\prime}(t)\right) d t+\int_{x}^{b} F\left(\gamma^{\prime}(t)\right) d t \\
& =\int_{a}^{b} F\left(\gamma^{\prime}(t)\right) d t=L(\gamma) .
\end{aligned}
$$

But, since $L(\gamma)=d(p, q)$ by part (1), this yields a contradiction.

6.6. Proposition. Suppose that the domain $D$ is Kobayashi simple and the Kobayashi metric $F$ is a complex Finsler metric. Let $\alpha<$ $0<\beta$. If $\gamma:[\alpha, \beta] \rightarrow D$ is a geodesic parametrized by arc length parameter $s$, then there is a holomorphic map $f: \Delta \rightarrow D$ such that

$$
\gamma(s)=f(\tanh (s)) \text {. }
$$

Moreover, the identity $f^{*} F=F_{\Delta}$ holds on $\tanh ([\alpha, \beta])$.

Proof. Since $F$ is a Finsler metric, Theorem 5.5 applies and there is an $\epsilon>0$ such that the restriction of $\gamma$ to the interval $[0, \epsilon]$ is 
the unique geodesic joining $p=\gamma(0)$ and $q=\gamma(\epsilon)$. Since $D$ is Kobayashi simple, we may assume $d^{*}(p, q)=d(p, q)$ by taking $\epsilon$ smaller if necessary.

Let $f$ be the extremal map joining $p$ and $q$ such that $f(0)=p$ and $f\left(b_{1}\right)=q$ for some $b_{1} \in[0,1)$. Then, by Lemma 6.1 , the curve $\sigma(t)=f(t)$ for $t \in\left[0, b_{1}\right]$ is also a geodesic joining $p$ and $q$. By the uniqueness of such a geodesic, $\gamma$ and $\sigma$ must define the same curve up to parametrization. Recall, from Lemma 6.1 again, that $f$ is an isometry between $F_{\Delta}$ and $F$ on $\left[0, b_{1}\right]$. Hence the arc length of the curve $\sigma(t)$ from $t=0$ to $t=x$ is $\delta(0, x)=\tanh ^{-1}(x)$ for $x \in\left[0, b_{1}\right]$. Thus, for $x \in\left[0, b_{1}\right]$, we have $\gamma\left(\tanh ^{-1}(x)\right)=f(x)$. Therefore, the identity (6.7) is proved for $s \in[0, \epsilon]$.

Consequently, the geodesic $\gamma(s)$ is real analytic for $s \in(0, \epsilon)$. Since the same argument can be applied to the curve $\gamma_{c}(s)=\gamma(s-c)$ for any $c \in(\alpha, \beta), \gamma(s)$ is real analytic on $(\alpha, \beta)$. Since both sides of the identity (6.7) are real analytic, the identity (6.7) should hold for all $s \in[\alpha, \beta]$.

To complete the proof, let $A=\sup \left\{x: f^{*} F=F_{\Delta}\right.$ on $\left.[0, x]\right\}$. Suppose $A<\tanh \beta$. Then we can take $x_{0}$ and $x_{1}$ such that $x_{0}<A<$ $x_{1}<\tanh \beta$ and there is a unique geodesic joining $\tilde{p}=f\left(x_{0}\right)$ and $\tilde{q}=f\left(x_{1}\right)$. By taking $x_{0}$ and $x_{1}$ closer if necessary, we may also assume that $d^{*}(\tilde{p}, \tilde{q})=d(\tilde{p}, \tilde{q})$. If $h \in D(\Delta)$ is an extremal map for $\tilde{p}, \tilde{q}$ such that $h\left(x_{0}\right)=\tilde{p}$ and $h\left(b_{2}\right)=\tilde{q}$ for some $b_{2}>x_{0}$ then, by Lemma 6.1, the curve $h(t)$ for $t \in\left[x_{0}, b_{2}\right]$ is a geodesic joining $\tilde{p}$ and $\tilde{q}$. Note that, if we let $s_{0}=\tanh x_{0}$ and $s_{1}=\tanh x_{1}$, then the curve $\gamma(s)$ for $s \in\left[s_{0}, s_{1}\right]$ is also a geodesic curve joining $\tilde{p}$ and $\tilde{q}$ :

$$
\gamma\left(s_{0}\right)=\gamma\left(\tanh x_{0}\right)=f\left(x_{0}\right)=\tilde{p}, \quad \gamma\left(s_{1}\right)=\gamma\left(\tanh x_{1}\right)=f\left(x_{1}\right)=\tilde{q} .
$$

Again, by the uniqueness of such a geodesic, the curves $h(t)$ and $\gamma(s)$ should define the same curve up to parametrization. By Lemma 6.1 again, we have $h^{*} F=F_{\Delta}$ on $\left[x_{0}, b_{2}\right]$, and hence the arc length of the curve $h(t)$ for $t \in\left[x_{0}, x\right]$ is $\delta\left(x_{0}, x\right)$. Therefore, parametrizing both curves by arc length, we obtain

$$
\gamma\left(\delta\left(x_{0}, x\right)+s_{0}\right)=h(x)
$$

for $x \in\left[x_{0}, b_{2}\right]$. (Note that the length $\delta\left(x_{0}, b_{2}\right)$ of the curve $h(t)$ defined for $t \in\left[x_{0}, b_{2}\right]$ is the length of the curve $\gamma(s)$ defined for $s \in\left[s_{0}, s_{1}\right]$. Hence, the identity $s_{1}-s_{0}=\delta\left(x_{0}, b_{2}\right)$ follows and this implies $b_{2}=x_{1}$.)

Observe that the identity (6.8) can be simplified to $\gamma(\tanh x)=h(x)$ since $\delta\left(x_{0}, x\right)+s_{0}=\tanh x-\tanh x_{0}+s_{0}=\tanh x$. Hence, combining 
this with the identity (6.7), we conclude that $f(x)=\gamma(\tanh x)=h(x)$ for $x \in\left[x_{0}, x_{1}\right]$. This implies that $f^{*} F=h^{*} F=F_{\Delta}$ on $\left[0, x_{1}\right]$ which contradicts the fact that $A<x_{1}$. Hence, we must have $A=$ $\tanh \beta$ and $f^{*} F=F_{\Delta}$ on $[0, \tanh \beta]$.

A similar method can be applied to show that the identity $f^{*} F=F_{\Delta}$ holds on $[\tanh \beta, 0]$.

6.9. Corollary. Suppose that D is Kobayashi simple and the Kobayashi metric $F$ of $D$ is a Finsler metric of class $C^{2}$. Then the following are true:

(1) Each point $p \in D$ has a neighborhood $U_{p}$ such that, for any $q \in U_{p}$, there is a unique extremal disk joining $p$ and $q$.

(2) Any geodesic curve of $F$ parametrized by arc length is (real) analytic.

Proof. Let $U_{p}$ be a neighborhood of $p$ such that for any $q \in U_{p}$, the geodesics joining $p$ and $q$ is unique and $d^{*}(p, q)=d(p, q)$. The uniqueness of extremal disk follows from Lemma 6.1 (2), the formula (6.7) and the analyticity of extremal maps. That $\gamma(t)$ is real analytic directly follows from the formula (6.7).

6.10. Definition [K]. A bounded domain $D$ is called complete if the Kobayashi distance $d$ is complete as a topological metric.

6.11. Theorem. Suppose that the Kobayashi metric $F$ on a complete Kobayashi simple domain $D$ is a Finsler metric. Then, the distance $d(p, q)$ between any two points $p, q \in D$, can be realized by the Poincaré distance on a single analytic disk joining $p$ and $q$ i.e. any $p, q \in D$ satisfies the condition

$$
d^{*}(p, q)=d(p, q) .
$$

In particular, $d^{*}$ satisfies the triangle inequality.

Proof. By Theorem 5.7, there is a geodesic (parametrized by arc length) $\gamma:[0, \beta] \rightarrow D$ with length $L(\gamma)=d(p, q)$ such that $\gamma(0)=p$ and $\gamma(\beta)=q$. By Proposition 6.6, there is a map $f \in D(\Delta)$ such that

$$
\gamma(s)=f(\tanh (s))
$$

where $s$ is the arc length and $f^{*} F=F_{\Delta}$ on $\tanh ([0, \beta])$. If we let $b=\tanh \beta$, we have

$$
f(0)=\gamma(0)=p, \quad f(b)=f(\tanh \beta)=\gamma(\beta)=p .
$$


Hence, we have

$$
\begin{aligned}
d^{*}(p, q) & \leq \delta(0, b)=\int_{0}^{b} F_{\Delta}\left(\left.\frac{\partial}{\partial x}\right|_{x=t}\right) d t=\int_{0}^{b} F\left(\frac{\partial f}{\partial x}(t)\right) d t \\
& =L(\gamma)=d(p, q) \leq d^{*}(p, q)
\end{aligned}
$$

and the theorem is proved.

\section{REFERENCES}

[Ba] T. J. Barth, The Kobayashi indicatrix at the center of a circular domain, Proc. Amer. Math. Soc.,, 88 (1983), 527-530.

[F] J. J. Faran, Hermitian Finsler metrics and the Kobayashi metric, J. Differential Geom.,, 31 (1990), 601-625.

[GF] I. M. Gelfand and S. V. Fomin, Calculus of Variations, Prentice-Hall, Englewood Cliffs, NJ, 1963.

[Gr] I. Graham, Boundary behavior of the Caratheodory and Kobayashi metrics on strongly pseudo-convex domains in $\mathbf{C}^{n}$ with smooth boundary, Trans. Amer. Math. Soc.,, 207 (1975), 219-240.

[K] S. Kobayashi, Hyperbolic Manifolds and Holomorphic Mappings, Marcel Dekker, New York, 1970.

[Ker] N. Kerzman, Taut manifold and domains of holomorphy in $\mathbf{C}^{n}$, Notices Amer. Math. Soc.,, 16 (1969), 675.

[Kr] S. Krantz, Convexity in complex analysis, Several Complex Variables and Complex Geometry, Proc. Sympos. Pure. Math., vol. 52, Amer. Math. Soc., Providence, RI, 1991, pp. 119-137.

[L1] L. Lempert, La métrique de Kobayashi et la représentation des domains sur la boule, Bull. Soc. Math. France,, 109 (1981), 427-474.

[L2] _ Intrinsic distances and holomorphic retracts, Complex Analysis and Applications,, 81 (1984), 341-364.

[MM] M. Matsumoto, Foundations of Finsler Geometry and Special Finsler Spaces, chapter VII, Kaiseisha Press, printed in Japan, 1986.

[R] H. L. Royden, Remarks on the Kobayashi Metric, Lecture Notes in Math., vol. 185, Springer, Berlin, 1971, pp. 125-137.

[S] S. Sternberg, Lectures on Differential Geometry, Prentice Hall, Englewood Cliffs, NJ, 1964.

[W] J. H. C. Whitehead, Convex regions in the geometry of paths., Quart. J. Math.,, 3 (1932), 33-42.

[Wu] H. Wu, Normal families of holomorphic mappings, Acta Math.,, 119 (1967), 193-233.

Received August 6, 1991.

WASHINGTON UNIVERSITY

ST. LouIs, MO 63130

Current address: Purdue University

West Lafayette, IN 47907 



\title{
PACIFIC JOURNAL OF MATHEMATICS
}

Founded by

\author{
E. F. BeCKenBACH (1906-1982) F. Wolf (1904-1989)
}

\section{EDITORS}

Sun-Yung A. Chang

(Managing Editor)

University of California

Los Angeles, CA 90024-1555

chang@math.ucla.edu

\section{F. Michael Christ}

University of California

Los Angeles, CA 90024-1555

christ@math.ucla.edu

Herbert Clemens

University of Utah

Salt Lake City, UT 84112

clemens@math.utah.edu
THOMAS ENRIGHT

University of California, San Diego

La Jolla, CA 92093

tenright@ucsd.edu

Nicholas ERCOLANI

University of Arizona

Tucson, AZ 85721

ercolani@math.arizona.edu

R. FINN

Stanford University

Stanford, CA 94305

finn@gauss.stanford.edu

VAUghan F. R. Jones

University of California

Berkeley, CA 94720

vfr@math.berkeley.edu
STEVEN KERCKHOFF

Stanford University

Stanford, CA 94305

spk@gauss.stanford.edu

Martin ScharLemanN University of California Santa Barbara, CA 93106 mgscharl@math.ucsb.edu

Harold Stark

University of California, San Diego La Jolla, CA 92093

V. S. VARADARAJAN University of California

Los Angeles, CA 90024-1555

vsv@math.ucla.edu

\section{SUPPORTING INSTITUTIONS}

UNIVERSITY OF ARIZONA

UNIVERSITY OF BRITISH COLUMBIA

CALIFORNIA INSTITUTE OF TECHNOLOGY

UNIVERSITY OF CALIFORNIA

UNIVERSITY OF MONTANA

UNIVERSITY OF NEVADA, RENO

NEW MEXICO STATE UNIVERSITY

OREGON STATE UNIVERSITY
UNIVERSITY OF OREGON

UNIVERSITY OF SOUTHERN CALIFORNIA

STANFORD UNIVERSITY

UNIVERSITY OF HAWAII

UNIVERSITY OF UTAH

WASHINGTON STATE UNIVERSITY

UNIVERSITY OF WASHINGTON 


\title{
PACIFIC JOURNAL OF MATHEMATICS
}

\author{
Volume $162 \quad$ No. $1 \quad$ January 1994
}

Semisimplicity of restricted enveloping algebras of Lie superalgebras $\quad 1$

JEFFERY MARC BERGEN

A classification of certain 3-dimensional conformally flat Euclidean hypersurfaces

OSCAR J. GARAY

Braided groups of Hopf algebras obtained by twisting

D. GUREVICH and SHAHN MAJID

Flat connections, geometric invariants and the symplectic nature of the 45 fundamental group of surfaces

K. GURUPRASAD

Spin models for link polynomials, strongly regular graphs and Jaeger's 57 Higman-Sims model

PIERRE DE LA HARPE

On the uniqueness of representational indices of derivations of $C^{*}$-algebras

EDWARD KISSIN

On infinitesimal behavior of the Kobayashi distance

MYUNG YULL PANG

Vertex operator construction of standard modules for $A_{n}^{(1)}$

MIRKO PRIMC

Bergman and Hardy spaces with small exponents

KEHE ZHU 